\title{
INVARIANTS OF A PORISTIC SYSTEM OF TRIANGLES*
}

\author{
BY J. H. WEAVER
}

1. Introduction. In the last quarter of a century considerable work has been done on various systems of triangles. $\dagger$ Several fixed circles have been discovered which are associated with poristic systems of triangles. However, so far as the writer knows, no attempt has been made to determine all the invariant curves of any degree for any system of triangles. The present paper determines the invariant curves of the first and second degrees for a poristic system of triangles which has a fixed incircle and a fixed circumcircle.

2. General Considerations. Let there be a triangle $A B C$ with a fixed incircle, center $I$, and a fixed circumcircle, center $O$. If we consider $O I$ as the $X$-axis and $I$ as the origin, the equations of the sides of the triangle may be written in the normal form as follows:

$$
\left\{\begin{array}{l}
B C: \quad x \cos A_{1}+y \sin A_{1}-r=0, \\
C A: \quad x \cos A_{2}+y \sin A_{2}-r=0, \\
A B: \quad x \cos A_{3}+y \sin A_{3}-r=0,
\end{array}\right.
$$

where $r$ denotes the radius of the incircle.

If $A^{\prime} B^{\prime} C^{\prime}$ be any other triangle of the system the equations of its sides are

$$
\left\{\begin{array}{l}
B^{\prime} C^{\prime}: x \cos A_{1}^{\prime}+y \sin A_{1}^{\prime}-r=0, \\
C^{\prime} A^{\prime}: \quad x \cos A_{2}^{\prime}+y \sin A_{2}^{\prime}-r=0, \\
A^{\prime} B^{\prime}: x \cos A_{3}^{\prime}+y \sin A_{3}^{\prime}-r=0 .
\end{array}\right.
$$

Let $P\left(x^{\prime}, y^{\prime}\right)$ be any point in the plane of the triangles. Then the distances of $P$ from the sides (1) and (2) are

$$
\begin{array}{ll}
\alpha_{i}=x^{\prime} \cos A_{i}+y^{\prime} \sin A_{i}-r, & (i=1,2,3), \\
\alpha_{i}^{\prime}=x^{\prime} \cos A_{i}{ }^{\prime}+y^{\prime} \sin A_{i}{ }^{\prime}-r, & (i=1,2,3),
\end{array}
$$

respectively.

* Presented to the Society, September 8, 1926.

† See Gallatley, Modern Geometry of the Triangle, second edition, London, 1910; and Coolidge, Treatise on the Circle and Sphere, Oxford, 1916. 
Eliminating $x^{\prime}$ and $y^{\prime}$ from (3) and (4), we obtain

$$
\begin{aligned}
\alpha_{i}^{\prime}=\frac{\left|\alpha_{1}, \sin A_{2}, 1\right|}{\left|\cos A_{1}, \sin A_{2}, 1\right|} & \cos A_{i}^{\prime} \\
& \quad+\frac{\left|\cos A_{1}, \alpha_{2}, 1\right|}{\left|\cos A_{1}, \sin A_{2}, 1\right|} \sin A_{i}^{\prime}-r .
\end{aligned}
$$

The angle $A_{2}^{\prime}$ determines $A_{1}^{\prime}$ and $A_{3}^{\prime}$. Hence $\alpha_{i}^{\prime}$ depends upon $\alpha_{i}$ and $A_{2}^{\prime}$, if $A_{1}, A_{2}$ and $A_{3}$ remain fixed.

Equations (5) then determine a one-parameter group of transformations, and by the Lie Theory of one-parameter groups we have for an invariant

$$
\sum_{i=1}^{3}\left[\frac{\partial \alpha_{i}^{\prime}}{\partial A_{2}^{\prime}}\right]_{A_{2^{\prime}=A_{2}}} \frac{\partial}{\partial \alpha_{i}} \equiv 0 .
$$

Equation (6) for the particular case represented by equation (5) becomes

$$
\begin{aligned}
\sum_{i=1}^{3}[- & \frac{\left|\alpha_{1}, \sin A_{2}, 1\right|}{\left|\cos A_{1}, \sin A_{2}, 1\right|} \sin A_{i} \frac{d A_{i}}{d A_{2}} \\
& \left.\quad+\frac{\left|\cos A_{1}, \alpha_{2}, 1\right|}{\left|\cos A_{1}, \sin A_{2}, 1\right|} \cos A_{i} \frac{d A_{i}}{d A_{2}}\right] \frac{\partial}{\partial \alpha_{i}} \equiv 0 .
\end{aligned}
$$

If we choose for the triangle $A B C$ the special position where $B$ lies on the line $O I$, and to the right of $I$, equation (6') will be much simplified without loss of generality. In fact it becomes

$$
\left\{\begin{array}{l}
{\left[\left(2 \alpha_{2}-\alpha_{3}-\alpha_{1}\right) \cos ^{2} \frac{B}{2} \cdot \frac{d A_{1}}{d A_{2}}\right.} \\
\left.+\left(\alpha_{1}-\alpha_{3}\right)\left(\sin \frac{B}{2}+1\right)\left(\sin \frac{B}{2}\right) \frac{d A_{1}}{d A_{2}}\right] \frac{\partial}{\partial \alpha_{1}} \\
+\left(\alpha_{3}-\alpha_{1}\right)\left(\sin \frac{B}{2}+1\right) \frac{\partial}{\partial \alpha_{2}} \\
+\left[-\left(2 \alpha_{2}-\alpha_{3}-\alpha_{1}\right) \cos ^{2} \frac{B}{2} \cdot \frac{d A_{3}}{d A_{2}}\right. \\
\left.+\left(\alpha_{1}-\alpha_{3}\right)\left(\sin \frac{B}{2}+1\right) \sin \frac{B}{2} \cdot \frac{d A_{3}}{d A_{2}}\right] \frac{\partial}{\partial \alpha_{3}} \equiv 0
\end{array}\right.
$$


where $d A_{3} / d A_{2}=d A_{1} / d A_{2}=R /(R-d)$, in which $R$ denotes the radius of the circumcircle, and $d=O I$.

3. A First Degree Invariant. Let

$$
l \alpha_{1}+m \alpha_{2}+n \alpha_{3}=0
$$

represent any line in the plane of the triangle $A B C$. If we apply the differential operator (7) to the line (8), we obtain

$$
\begin{array}{r}
{\left[\left(k^{\prime}-k\right) l-k^{\prime \prime} m+\left(k^{\prime}+k\right) n\right] \alpha_{1}+(2 k l-2 k n) \alpha_{2}} \\
+\left[-\left(k^{\prime}+k\right) l+k^{\prime \prime} m-\left(k^{\prime}-k\right) n\right] \alpha_{3} \equiv 0
\end{array}
$$

where

$$
\begin{aligned}
k & =\cos ^{2} \frac{B}{2} \cdot \frac{d A_{1}}{d A_{2}} \\
k^{\prime} & =\left(\sin \frac{B}{2}+1\right) \sin \frac{B}{2} \cdot \frac{d A_{1}}{d A_{2}} \\
k^{\prime \prime} & =\sin \frac{B}{2}+1
\end{aligned}
$$

In order that (9) shall be satisfied we must have

$$
\left\{\begin{aligned}
\left(k^{\prime}-k\right) l-k^{\prime \prime} m+\left(k^{\prime}+k\right) n & =0, \\
l-n & =0, \\
-\left(k^{\prime}+k\right) l+k^{\prime \prime} m-\left(k^{\prime}-k\right) n & =0 .
\end{aligned}\right.
$$

The matrix of the set of equations (10) is of rank 2, hence there is one solution for $l, m, n$, other than zeros. A solution may then be written $l=l, m=\left(2 k^{\prime} / k^{\prime \prime}\right) l, n=l$. But $2 k^{\prime} / k^{\prime \prime}=1$ and the equation of the single invariant line of the system is

$$
\alpha_{1}+\alpha_{2}+\alpha_{3}=0 \text {. }
$$

This is the equation of the line in which the bisectors of the exterior angles of the triangle meet the opposite sides; hence we may state the following theorem.

THEOREM I. In a poristic system of triangles, which has a fixed incircle and a fixed circumcircle, the line determined by the points of intersection of the bisectors of the exterior angles of the triangle with the opposite sides is a fixed line. 
4. Second Degree Invariants. The equation of any curve of the second degree in the plane of the triangle $A B C$ may be written

(11) $l \alpha_{1}^{2}+m \alpha_{2}^{2}+n \alpha_{3}^{2}+2 p \alpha_{1} \alpha_{2}+2 s \alpha_{1} \alpha_{3}+2 t \alpha_{2} \alpha_{3}=0$.

If we apply the differential operator (7) to the equation (11) we obtain

$$
\begin{aligned}
& {\left[l \alpha_{1}+p \alpha_{2}+s \alpha_{3}\right]\left[\left(2 \alpha_{2}-\alpha_{3}-\alpha_{1}\right) k+\left(\alpha_{1}-\alpha_{8}\right) k^{\prime}\right] } \\
+ & \left(m \alpha_{2}+p \alpha_{1}+t \alpha_{3}\right)\left(\alpha_{3}-\alpha_{1}\right) k^{\prime \prime} \\
+ & {\left[-\left(2 \alpha_{2}-\alpha_{3}-\alpha_{1}\right) k+\left(\alpha_{1}-\alpha_{3}\right) k^{\prime}\right]\left[n \alpha_{3}+s \alpha_{1}+t \alpha_{2}\right] } \\
& \equiv 0 .
\end{aligned}
$$

In order that equation (12) shall be valid we must have the following set of equations.

$$
\begin{gathered}
l\left(k^{\prime}-k\right)-p k^{\prime \prime}+s\left(k^{\prime}+k\right)=0, \\
p=t, \\
n\left(k^{\prime}-k\right)-t k^{\prime \prime}+s\left(k^{\prime}+k\right)=0, \\
m k^{\prime \prime}-2 n k-p\left(k^{\prime}+k\right)+2 s k-t\left(k^{\prime}-k\right)=0, \\
l\left(k^{\prime}+k\right)-n\left(k^{\prime}+k\right)-p k^{\prime \prime}+t k^{\prime \prime}=0, \\
2 l k-m k^{\prime \prime}+p\left(k^{\prime}-k\right)-2 k s+\left(k^{\prime}+k\right) t=0 .
\end{gathered}
$$

The matrix of equations (13) is of rank 4. Hence there is a single infinity of solutions for the set of equations. This set of solutions may be written in terms of $l$ and $p$, as follows:

$$
n=l, t=p, \quad s=\frac{R-d}{R} p+\frac{d}{R} l, m=\frac{R+d}{R} l-\frac{d}{R} p .
$$

Equation (12) may therefore be written in the form

$$
\begin{aligned}
l \alpha_{1}^{2} & +\left(\frac{R+d}{R} l-\frac{d}{R} p\right) \alpha_{2}^{2}+l \alpha_{3}^{2}+2 p \alpha_{1} \alpha_{2} \\
& +2\left(\frac{R-d}{R} p+\frac{d}{R} l\right) \alpha_{1} \alpha_{3}+2 p \alpha_{2} \alpha_{3}=0 .
\end{aligned}
$$


By finding the polar of the incenter with respect to the family (14) we obtain a result which may be stated in the following theorem.

THEOREM II. The first polar of the incenter with respect to the family (14) is the line $\alpha_{1}+\alpha_{2}+\alpha_{3}=0$.

Also if we write down the condition that the system (14) shall represent two lines we find that it is necessary that $l=p \cdot *$

A substitution of this condition in (14) gives $\left(\alpha_{1}+\alpha_{2}+\alpha_{3}\right)=0$. Hence the straight lines of the system (14) consist of the first degree invariant counted twice.

If we set down the condition that equation (14) represent a circle we find

$$
p=l\left(\frac{R+d}{R}\right)
$$

A substitution of this condition in (14) gives for the equation of the circle the expression

$$
\begin{aligned}
& \alpha_{1}^{2}+\frac{2 r}{R} \alpha_{2}^{2}+\alpha_{3}^{2}+2\left(\frac{R+d}{R}\right) \alpha_{1} \alpha_{2} \\
& \quad+2\left(\frac{2 r+d}{R}\right) \alpha_{1} \alpha_{8}+2\left(\frac{R+d}{R}\right) \alpha_{2} \alpha_{3}=0 .
\end{aligned}
$$

The center of this circle lies on $O I$. And since we have chosen as our triangle of reference the triangle whose vertex $B$ lies on the line $O I$, the equation of $O I$ is

$$
\alpha_{1}-\alpha_{3}=0 \text {. }
$$

Also from the triangle itself we have

$$
\alpha_{1}+\left(\frac{R-d}{R}\right) \alpha_{2}+\alpha_{3}=\left(\frac{R-d}{R}\right)(R+d+r) .
$$

\footnotetext{
* See Whitworth, Modern Analytical Geometry, p. 244.
} 
If we solve equations (15), (16), (17) for $\alpha_{2}$ we find

$$
\alpha_{2}=\frac{d(R+d+r) \pm[R d(R+d)(R+d+r)]^{1 / 2}}{d} .
$$

The half sum of the two values of $\alpha_{2}$ gives the distance of the center of the circle (15) from the side $A C$ of the triangle. This distance is $R+d+r$. Hence we have the following theorem.

TheOREM III. The center of the invariant circle (15) lies on the circumcircle of the variable system of triangles.

The half difference of the values of $\alpha_{2}$ gives the radius $\rho$ of the circle (15). Consequently

$$
\rho=\frac{[R d(R+d)(R+d+r)]^{1 / 2}}{d} .
$$

Using this result we may easily prove the following theorem.

THEOREM IV. The line $\alpha_{1}+\alpha_{2}+\alpha_{3}=0$ is the radical axis of the incircle and the circle (15).

Ohio State University 